\title{
Railway infrastructure asset management: the whole-system life cost analysis
}

Dovile Rama and John D Andrews

Nottingham Transportation Engineering Centre, Faculty of Engineering, University of Nottingham, University Park, Nottingham, NG7 2RD, UK.

E: dovile.rama@nottingham.ac.uk, john.andrews@nottingham.ac.

\begin{abstract}
Delivering the railway infrastructure whose functionality is sustainable and uncompromised in terms of safety and availability under ever decreasing budget constraints is a great challenge. The successful accomplishment of this task relies on the effective management of individual assets within a wider whole system perspective. This is a highly complex decision making task where mathematical models are required to enable well informed choices.
\end{abstract}

In this paper a novel modelling framework is proposed for performing the whole system lifecycle cost analysis. The framework is based on two models: railway network performance and costs. Using the former model investigations of the effects of decisions can be carried out for the individual asset and the whole system. A Petri Net modelling technique is used to construct the model which is then analysed by means of Monte Carlo simulations. The infrastructure performance model is then integrated with the cost model to perform the lifecycle cost analysis.

A superstructure example is presented to demonstrate the application of the approach. The results show that taking into account interdependencies among the intervention activities greatly influences, not only the performance of the infrastructure, but also its lifecycle costs and thus should be included in the cost analysis. Thus, the proposed models enable more detailed and accurate economic forecast.

Keywords: railway infrastructure, asset management, Petri Nets, life cycle costs, system model.

\section{Introduction}

The management of the railway assets involves a range of activities such as building, inspection, maintenance, enhancement and renewal aimed at optimising performance, risks and costs of the infrastructure. This is a highly complex decision making task as various trade-offs need to be made 
and, importantly, interdependencies among the timing and choices of activities for multiple assets need to be taken into account in order to achieve the required levels of safety and availability at the lowest possible costs in a sustainable manner. Therefore modelling techniques based on cost (risk) analysis have been increasingly used as decision support solutions. In particular, techniques supporting long term assessment of decisions, such as life cycle cost (LCC) analysis, have attracted increasing attention from practitioners in recent years [1]. The LCC models have varying degrees of sophistication and can range from high level models aimed at forecasting work volumes and expenditure for a portfolio of assets to the models providing detailed lifecycle cost analysis of intervention regimes for a particular asset $[2,3,4,5,6]$.

Despite the fact that significant progress has been made in adopting models in the decision making process, there remains many challenges to be addressed in the development of effective asset management decision support tools. The key challenges in the railway industry in particular include $[7,8]:$

- Defining the configuration and granularity of the railway infrastructure that is sufficient to support the analysis at the level of detail required for the decision making.

- Defining factors influencing the lifecycle costs as well as their relationships. For example, the driving factor causing maintenance is degradation. Asset degradation in turn is influenced by the design and operational conditions of the infrastructure as well as the maintenance strategy adopted.

- Addressing the railway system-wide implications of the decisions. Activities performed on one asset group (e.g. track) may impact on another part of the railway system (e.g. signalling). Prioritization and clustering of activities among different asset groups can improve infrastructure performance (e.g. reduce downtime) and reduce maintenance costs (e.g. track possession costs).

- Dealing with uncertainty due to the stochastic nature of the model variables and parameters. For example, two levels of uncertainty are associated with the LCC approach. The first level of uncertainly pertains to the variation of unit costs over time (e.g. man hour cost) and costs associated with the infrastructure functionality (e.g. cost of delay minutes). Another level of uncertainty originates from predictions in the levels of costs mainly due to degradation and failure of assets and the resulting intervention needs. 
A novel modelling framework for evaluating multi-asset infrastructure LCC is presented in this paper. It has been developed to support asset management decisions in a whole-system context, thus allowing interdependencies among management decision of multiple assets to be considered both from the cost and performance perspectives. The framework proposed has been designed to address the problematic issues mentioned and presents the following advantages:

- The infrastructure model is constructed based on a hierarchical representation of the railway network, thus the analysis can be performed at various levels of granularity ranging from an individual maintainable item to a large multi-asset network.

- Degradation and maintenance models of each maintainable item are stochastic. Model parameters are determined considering asset degradation and/or intervention history, infrastructure utilization levels and specific intervention strategies.

- A modular modelling architecture is adopted meaning individual maintainable item models can be assembled into a single infrastructure system model. This way a multi-asset infrastructure model can be constructed taking into account asset interrelationships.

- A Petri Net (PN) modelling technique is used to construct the model is then analysed using Monte Carlo simulations. This modelling technique enables construction and analysis of multiasset infrastructure models of varying degree of complexity and detail.

The rest of the paper is organized as follows. First the concepts of the proposed asset management modelling framework are discussed. The application of the framework is demonstrated with a case study next. Finally, the paper finishes with the summary and conclusions section.

\section{Modelling framework for lifecycle cost assessment}

The generic framework for modelling infrastructure lifecycle costs consists of two major elements namely the infrastructure state model and the cost model (Figure 1). Using the state model, performance parameters are estimated considering the effects of changes in individual assets on other assets and subsequently on the infrastructure as a whole. The outputs of the infrastructure state model are then fed into the costs model to evaluate the life cycle costs. 


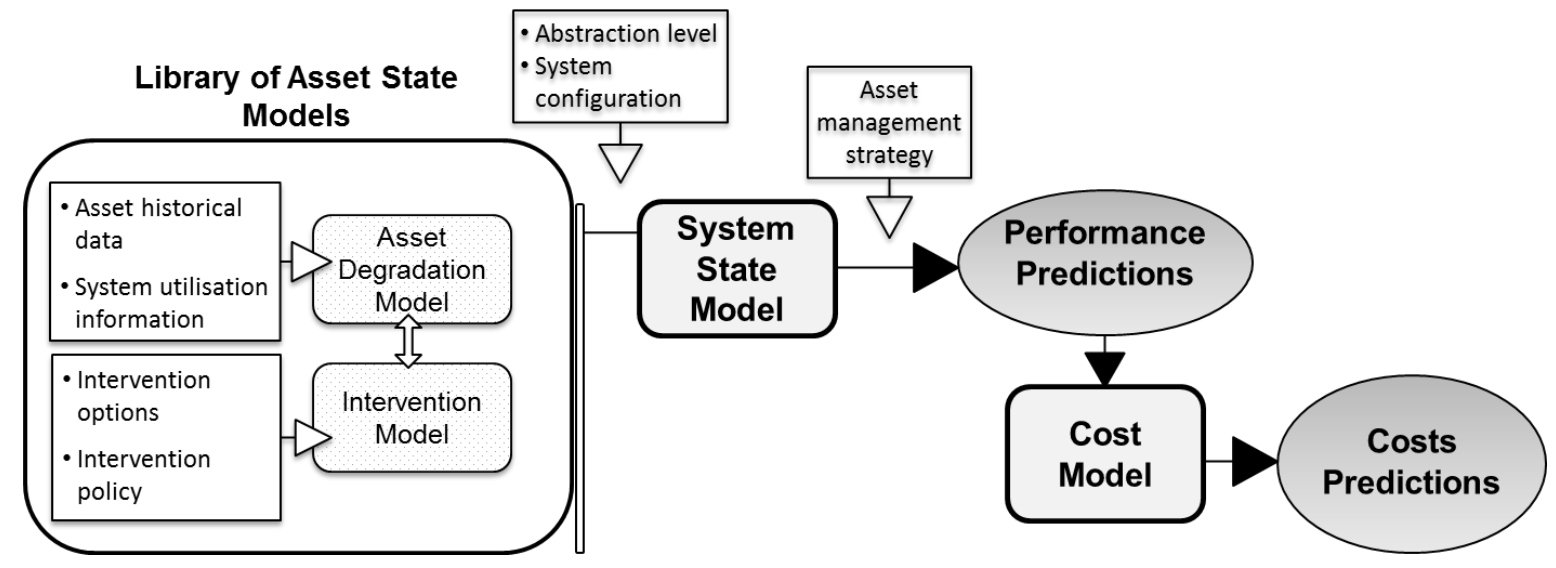

Figure 1. Overview of the proposed modelling framework for decision support in infrastructure asset management.

\subsection{Infrastructure state model}

\section{Model architecture}

The infrastructure state model has a hierarchical modular architecture allowing a multi-asset configuration of the infrastructure within a hierarchical topology of network to be depicted. The network topology consists of several levels. Network segments are at the lowest (bottom) level. A segment represents the smallest unit size of the track for which the degradation and intervention processes can be determined. A section which is formed from several segments is at the second hierarchical level. The section normally represents a longer portion of the track, e.g. from one junction to another. A number of joined sections then form a line. Lines which represent portions of the network between two major locations, e.g. major railway stations, are at the forth hierarchical network level. Operational routes are modelled at the next, i.e. fifth, level. Each of them comprises a specified number of lines and represents a railway network part in a specified region of the country. Finally, the whole network represents the top hierarchical level.

In terms of infrastructure assembly, individual maintainable items (assets), which have both degradation mechanisms and intervention strategies determined, are at the lowest indenture level. Two assets will be considered in this study, namely sleepers and rails. Higher indenture levels of the infrastructure assembly, e.g. track superstructure, are formed by assembling individual assets. The process is carried out until the indenture level of interest is achieved. Two indenture levels will be considered in the paper, namely individual asset and superstructure levels. 
Thus, in the presented hierarchical topology each individual asset is first modelled at a segment level. An individual asset state model is built for a specific asset to simulate its state changes over the time horizon. Every asset segment model is constructed by integrating degradation-failure and intervention processes. The degradation of the asset condition is modelled by using a finite number of discrete degradation states which are reached sequentially as the condition of the asset worsens. In order to take into account the phenomenon of variability in the degradation rates that exists even among the same types of assets, times to reach each degradation state are governed by appropriate stochastic distributions. The asset model includes options to account for specific inspection, testing, servicing, repair, renewal and upgrade alternatives. The choice of the activities and their timing can be based on various criteria including risk, asset condition, asset reliability or simply a predefined time regime which is determined by the management strategy chosen.

The asset segment models are then used as building blocks to construct state models for higher network hierarchical and/or infrastructure indenture levels. For example, in order to build a rail model for a single section, individual rail segment models will be integrated taking into account any requirements for coordination of intervention activities among multiple rail segments. Similarly, by joining models representing individual sections, a line, a route and finally the whole network model is constructed. In the same manner, a multi-asset (higher indenture level) infrastructure state model can be constructed. In this case, the integration process requires addressing interdependencies among intervention activities of different assets, common inspection routines as well as concurrent and opportunistic maintenance activities.

\subsection{Modelling technique: Petri Nets}

Petri Nets (PNs) [9] have been chosen as the modelling technique to be used due to its capability of modelling both stochastic and deterministic events. Furthermore, PNs can support the modular architecture of the infrastructure state model. Using the PN technique, individual PN models can be simply joined into a larger PN model, while, for example, the Markov method would require a new model to be constructed.

A standard PN consists of a set of places, a set of transitions and a set of directed arcs which connect places to transitions and vice versa. Places, depicted as circles in the PN, represent a state, 
precondition or post conditions of a specific activity or process being modelled. Transitions, depicted as bars in the PN, represent events or processes that result in changes in the state or condition. In order to depict the dynamics of the changes tokens are used. Tokens reside in places signifying that the current state or condition associated with that place is true. Tokens move from place to place through associated transitions according to transition firing rules. A transition becomes enabled for firing when all its input places are marked, i.e. the number of tokens each place contains is at least equal to the arc multiplicity going from the place to the transition. The enabled transition fires by removing as many tokens as the multiplicity of the input arcs from each input place, and adding as many tokens as the multiplicity of the output arcs to each output place. The firing of the transition can happen instantaneously or it can be delayed for a certain time period. Transitions used in the infrastructure state model can have deterministic, random or probabilistic firing delay times. A more detailed description of the standard PN technique can be found in [9] and [10].

In the model presented in the paper an extended PN formalism has been used. Extensions introduced include an inhibit arc [9], a reset transition, a place condition transition, a decision-making transition, a periodical transition and a multi-functional transition [11].

Analysis of a system, particularly one modelled using a large and complex PN (as in the case of the model presented here), is commonly performed by means of simulations and the discrete event simulation technique has been chosen for the simulation of PNs in this study. Furthermore, when PNs model stochastic events each simulation produces a different set of parameter values of the modelled system. Thus the Monte Carlo simulation approach has been employed where simulation experiments are repeated by sampling stochastic parameters from appropriate distributions to produce a large statistical sample of performance parameters from which statistical results can be obtained.

Each simulation experiment mimics the behaviour of the modelled system over a specified time horizon and produces two types of outputs that facilitate the assessment of the system: 1) the number of tokens residing in specified places and 2) the duration the tokens reside in the specified places over the modelled time horizon. By counting the number of tokens received by the place over the entire simulation period, parameters such as numbers of failures or maintenance activities performed are evaluated. Similarly, by accumulating time periods when the place remains marked over the entire simulation period, time related performance parameters, e.g. mean time to failure, are obtained. 
Due to the enhanced features introduced to the PN technique, commercial software could not be used and special $\mathrm{C} / \mathrm{C}++$ based software had to be developed for the simulation and analysis of PN models.

\subsection{Lifecycle Cost Model}

In very simple terms LCC represents an expenditure profile over a specified period of time. BS EN 60300 [12] provides a framework for a systematic and orderly approach to a breakdown of costs incurred over the lifecycle, which can be tailored to the scope and the purpose of a specific LCC study. It involves identification of individual LCC cost elements and requires the breakdown of the system to lower indenture levels, cost categories (e.g. labour, materials) and life cycle phases to be specified. The cost elements are then aggregated to form the LCC in accordance with cost breakdown structure.

The LCC model used in the study has been developed by adopting the methodology proposed in [12]. Two indenture levels will be considered, namely the superstructure and individual assets (sleepers and rails). Figure 2 presents the cost breakdown where cost categories are identified with a dashed line. All of the cost categories considered in the model represent annual costs, except infrastructure renewal costs which are estimated for individual cases. Cost elements are then defined by pairing up each item (asset, superstructure) with each cost category appropriate for the item in question.

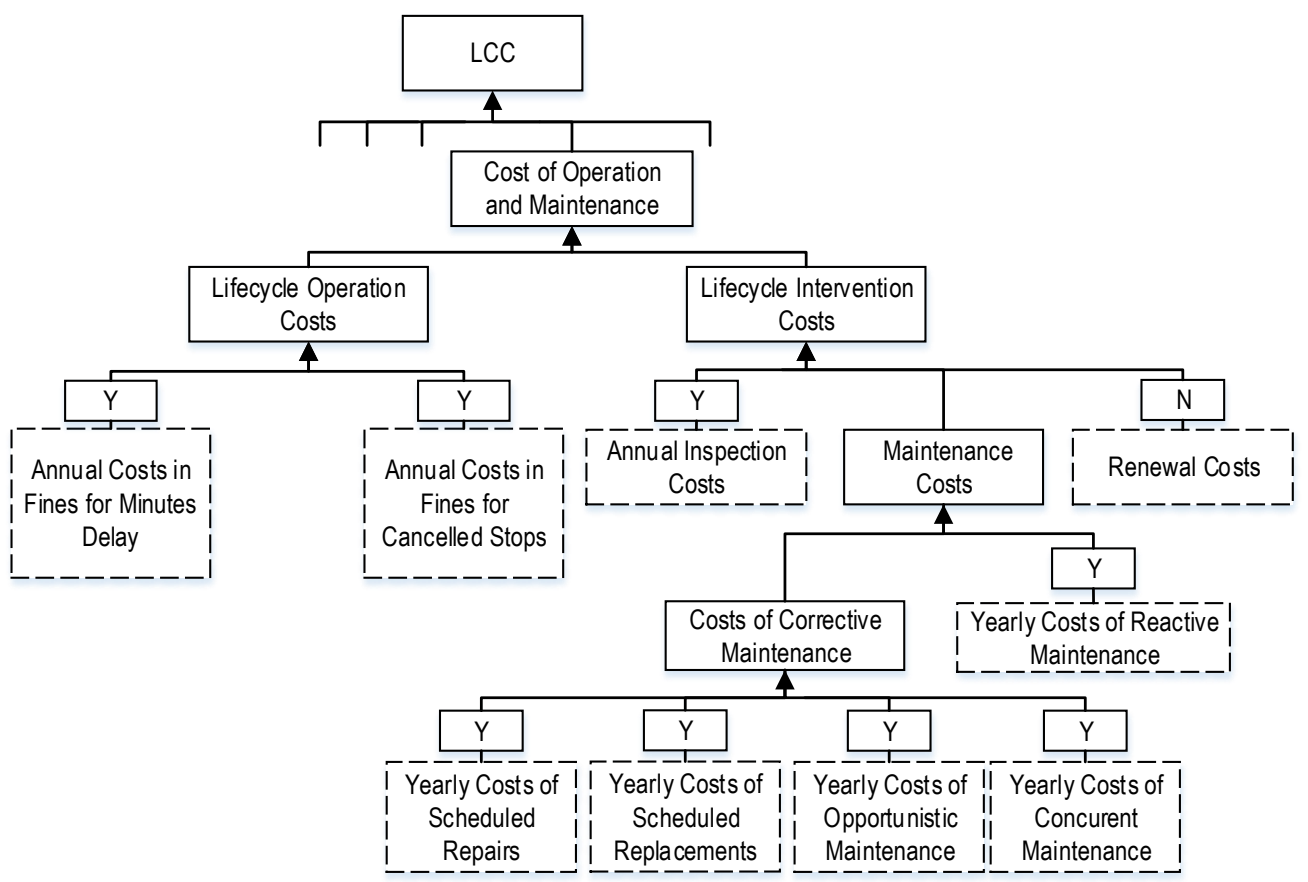

Figure 2. LCC breakdown structure. 
Only the intervention and operation costs will be considered in the case study. Annual intervention costs are subdivided into three cost constituents: labour, materials and equipment costs. The estimates of the cost elements which are associated with the intervention cost categories are calculated by summing the three constituents:

$$
C_{j}^{a, c}=n_{j}^{E, a, c} E_{j}^{a, c}+t_{j}^{L, a, c} L_{j}^{a, c}+n_{j}^{M, a, c} M_{j}^{a, c}
$$

Here $C_{j}^{a, c}$ denotes the costs related to a cost element associated with an asset a and a cost category $c$, acquired over the jh year, $n_{j}^{E, a, c}$ is the number of shifts performed, $t_{j}^{L, a, c}$ is the total duration of the shifts and $n_{j}^{M, a, c}$ is the amount of materials used for the intervention activities carried out. Accordingly, $E_{j}^{a, c}$ is a price of equipment per single shift, $L_{j}^{a, c}$ is a price of a single man-hour and $M_{j}^{a, c}$ is a unit price of materials used for the intervention activities.

The operational performance costs include fines paid by the infrastructure owner for Minutes Delay and Cancelled Stops caused by incidents attributed to infrastructure problems. Under the UK rail performance regime Minutes Delay and Cancelled Stops are the main operational performance parameters. Cost elements associated with the operational performance costs in a specified part of the network denoted as $i$ are estimated using the following equation:

$$
C_{j}^{i, c}=t_{j}^{i, D} D^{i}
$$

where $t_{j}^{i, D}$ is the aggregate of minutes of lateness beyond train targets in the th year and $D^{i}$ is a fine paid for one minute of lateness.

The lifetime costs of a cost category $c$ are estimated by:

$$
C^{c}=\sum_{j=1}^{Y} \frac{[\mathbf{I}] \cdot\left[\mathbf{c}_{j}^{c}\right]^{T}}{(1+r)^{j}}
$$

where $\left[\mathbf{C}_{j}^{c}\right]^{T}$ is a transpose of vector $\mathbf{C}_{j}^{c}$ whose components are cost estimates of cost elements associated with cost category $c, I$ is the identity matrix, $Y$ is the life period of infrastructure analysed expressed in years and $r$ is a discount rate. 
All prices and cost units used for the estimation of different cost elements are provided as input data in the model. Other parameters, such as the number of maintenance shifts or their duration, are obtained from the infrastructure sate model.

The LCC model with the cost categories proposed, differently to a popular way of simply aggregating intervention costs of individual assets [7, 13], takes into account reductions achieved through consolidation of intervention activities using either opportunistic or concurrent maintenance strategies.

\section{Case study}

\subsection{Superstructure PN Model}

In the case study presented a track superstructure on a single track railway line is analysed. For modelling purposes, the line which is 50 miles in length is divided into 5 sections each one comprising of $801 / 8$ th mile homogeneous segments. The superstructure is modelled as a multi-component system comprising of sleepers and rails.

In the PN models presented places and transitions are identified with a unique code (name) which, if needed, can also identify a specific position (i.e. a segment and/or section number) within the hierarchical structure of the network. Place names start with "P" and accordingly transition names start with "T". Furthermore, by using particular style or colour of PN object specific attributes of nodes are identified. For example, dashed lines (arcs) are used to link conditional input places to place conditional transitions. An arc with arrows on both sides represents two arcs of opposite directions between the same transition and place. A grey rectangle represents an immediate transition. Some of the attributes of transitions are also presented textually. For example, a transition symbol with a letter "t" signifies that the transition has a deterministic firing delay time. Rectangles with "P", "DM" and "PC" represent periodic, decision making and place conditional transitions respectively.

\section{Asset Model}

The superstructure line PN model is broadly based on the PN model initially presented in [11]. Therefore here only a brief description of the PN model will be provided. First the asset segment models are introduced. The degradation process of the two assets is gradual and is modelled using five discrete asset condition states (presented as places in the PN). Each state represents a 
degradation severity level identified by the urgency of the intervention needed in order to prevent further deterioration of the asset condition. It ranges from a very good state, where an intervention is not required, to a very poor, where an intervention is immediate. Durations that assets spend in each state before moving to the next one are stochastic and their values are sampled from appropriate statistical distributions which in this case are assumed to be exponential. Parameters of the distributions were estimated by performing a statistical analysis of data obtained from the asset maintenance database and the rail defect management system. An example of the sleepers' degradation sub-net is shown in Figure 3.

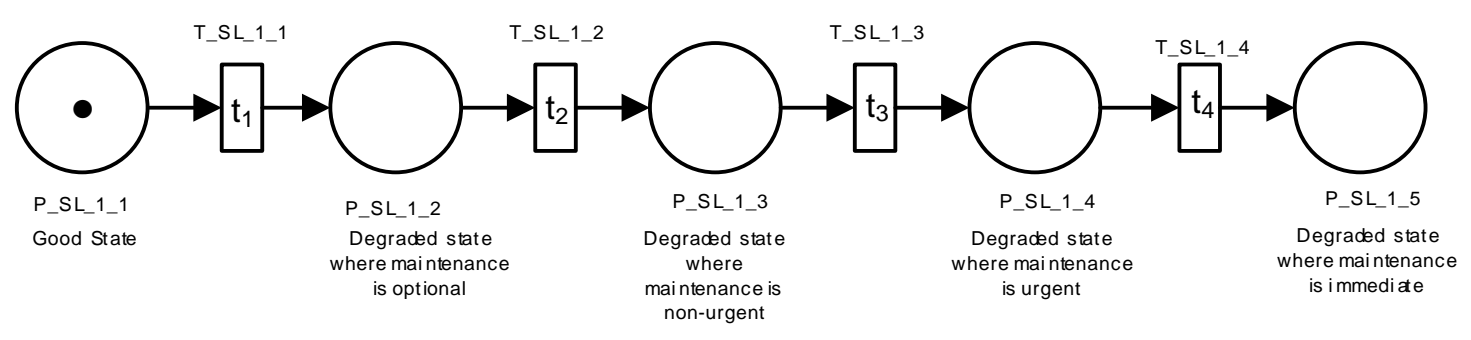

Figure 3. Sleepers Degradation Sub-net

Railway assets are inspected on a regular basis in order to detect any signs of degradation or faults. Arrangements for the inspection of superstructure components are specified as part of the permanent way inspection regime based on the category of the track [14]. For the track category considered in the case study, visual superstructure inspections are carried out every 2 weeks by walking along the tracks and noting down defects. Rails are inspected with the ultrasonic test unit (UTU) once every 4 weeks.

To improve the efficiency of the model only inspections during which changes in asset condition are detected are modelled. In Figure 4 a typical PN sub-net is presented modelling the inspection of sleepers and the detection of the state where defect elimination is not urgent. Here a periodic transition (T_SL_2_2) is used to determine the time of the next scheduled inspection during which deterioration in the condition of the sleepers in a particular segment will be detected. The firing delay time of the transition signifies the time period from the change in the asset's condition till the next scheduled inspection and is equal to the remainder after division of the system lifetime by the inspection period (in this case 14 days). As a result of firing the transition places P_SL_1_3 and P_SC_2_1 will become marked subsequently enabling transition T_SL_1_6. This is a decision 
making transition which has conditional place marking rules associated with it to ensure that only a single (the most severe) condition degradation state is identified in a segment at a time. The new marking of places obtained after immediately firing the transition signifies that a new degradation state of sleepers in the segment in question has been detected and, hence, maintenance has been requested. Subnets modelling the detection of other states are constructed in the same manner.

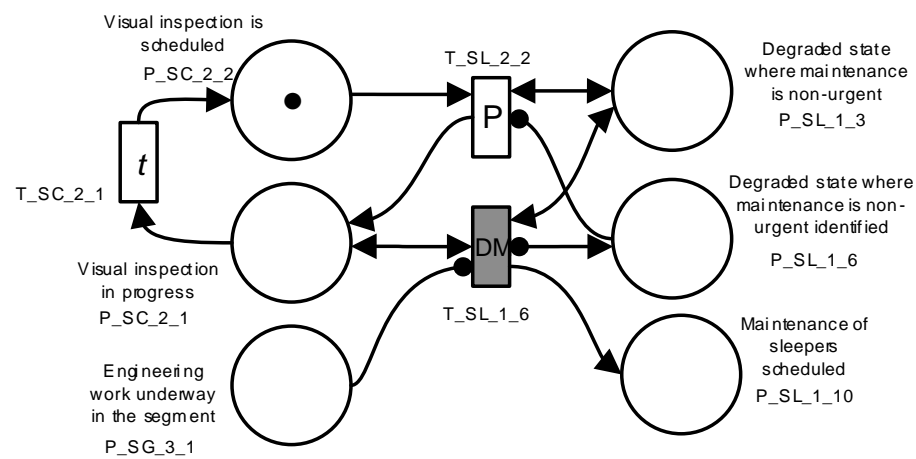

Figure 4. Sub-net modelling visual inspection activities.

A range of maintenance activities is modelled in the case study. Reactive maintenance of sleepers involves either repairs of defective, e.g. incorrectly spaced, sleepers or replacement of ineffective sleepers. Reactive interventions of rails involve either rail grinding, welding or rail replacement. Some of the rail maintenance activities are used more often than the others for specific rail conditions. In the model, historical intervention data is used to estimate the likelihood of the possible choices.

The sleepers' maintenance PN sub-net is shown in Figure 5 as an example. Both delays in the intervention activities and the intervention duration times depend on the degradation severity of the asset condition. The times are assumed to follow lognormal distributions whose parameters are determined based on track maintenance guidance and requirements [15].

\section{System Model}

The superstructure line PN model is constructed by first building the superstructure section models which are then used to construct the line model. The superstructure section PN is constructed by replicating the sleepers and rails segment PNs 80 times and subsequently integrating them.

Specifically, since the model assumes that inspection schedules are prepared for individual sections, each sleeper segment PN sub-net, modelling the inspection of the asset in that segment need to be 
linked to the PN sub-net modelling the scheduling of visual inspections in the corresponding section. Accordingly, rail segment inspection sub-nets need to be linked to the corresponding rail inspection sub-net.

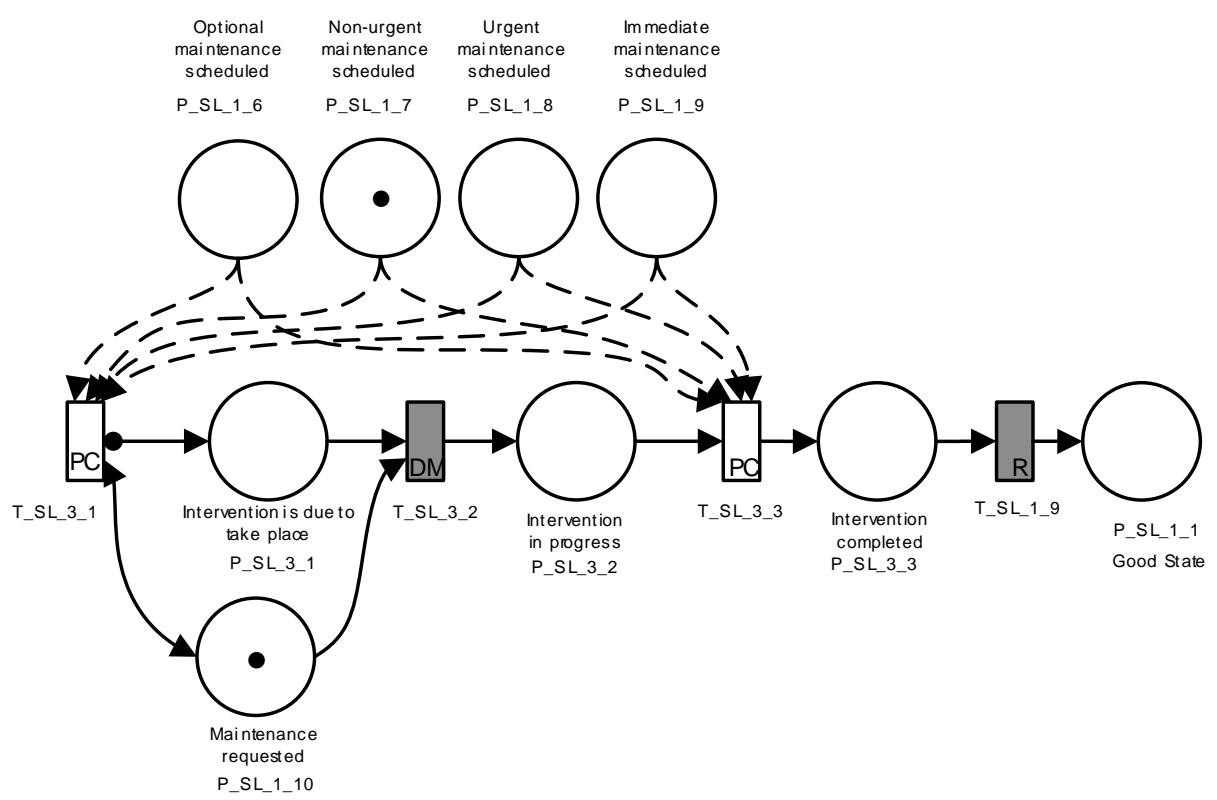

Figure 5. Sleepers intervention PN sub-net.

For the analysis purposes, several new places can also be added when joining the segment models to enable the monitoring of the superstructure performance within the section. For example, a single place can be added to represent the introduction of an emergency speed restriction (ESR) for the section. The place will be linked to each segment degradation sub-net and will receive a token every time any of the places in the segment models representing the state where ESR is required becomes marked. Similarly, by introducing an additional place section closures to rail traffic can be modelled.

Having constructed a multi-segment superstructure section PN and introducing several additional nodes allows modelling of opportunistic and concurrent asset maintenance strategies. By using the opportunistic maintenance strategy, the maintenance of the asset can be carried out earlier than planned taking advantage of the section possession time scheduled for the maintenance of another asset in the specified segment. To implement opportunistic maintenance scenarios for sleepers, for instance, transition labelled T_SL_3_3 in the intervention PN sub-net (Figure 5) is a decision making transition, hence having an enhanced functionality. Following the firing of the transition, places in the PNs of specified adjacent segments representing the initiation of maintenance ahead of the planned 
schedule will be marked based on the additional marking rules introduced with the transition. As a result, transitions labelled T_SL_3_1 in the corresponding segments will become enabled and fire instantly. Similarly, to model concurrent maintenance of assets, e.g. simultaneous replacement of rails and ineffective sleepers in the same segment, specific additional marking rules can be introduced to the corresponding decision making transition in the segment PNs of the assets.

The superstructure line PN model will be constructed by replicating and integrating five section PNs. Similar to the section PN, additional places can be introduced in the line model enabling the monitoring of the superstructure performance parameters at the line level. These places will be linked to every segment PN.

\subsection{Superstructure Cost Model}

Cost elements used in the case study are defined for the whole superstructure and individual assets as presented in Table 1.

\section{Table 1. Cost Elements}

\begin{tabular}{|c|c|c|c|c|c|c|c|}
\hline & $\frac{\infty}{\frac{\pi}{0}}$ & 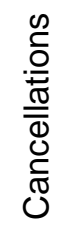 &  & 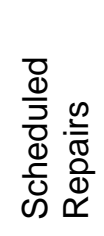 & 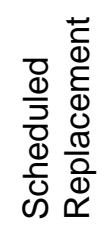 & 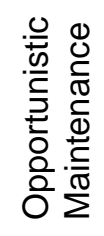 & 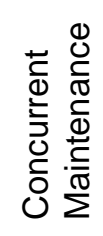 \\
\hline Superstructure & $x$ & $x$ & $\mathrm{x}$ & & & & \\
\hline Sleepers & & & & $\mathrm{x}$ & $\mathrm{x}$ & $\mathrm{x}$ & $\mathrm{x}$ \\
\hline Rails & & & & $x$ & $x$ & & \\
\hline
\end{tabular}

The specified cost elements are used for calculations of the LCC by employing Equations 1-3. First, costs of each category are calculated for every year of the time period considered. The expenditure in fines due to delays and cancellations caused by incidents is calculated for the superstructure as a whole. For this purpose Equation 2 is used where the rate for every minute of lateness is equal to $£ 35$. Even though penalty rates differ widely, in practice, this is an average rate paid by the infrastructure owner. The total number of minutes of train delays for each year is obtained from the simulations of the superstructure state model.

Yearly inspection costs are calculated by aggregating costs of visual inspection and UTU inspection activities carried out over the entire length of the line. For the calculations the costs of visual and UTU 
inspections were estimated to be $£ 70$ and $£ 250$ per mile respectively. Maintenance costs are calculated for each asset category individually including costs of scheduled repairs, scheduled replacements, opportunistic maintenance, concurrent maintenance and reactive maintenance activities by using equation 1 . Theoretical equipment, labour and material costs were used to estimate the intervention costs. The quantities of equipment and materials used and man-hours spend for activities over each year are obtained from the simulation of the superstructure state model. The calculated asset yearly intervention costs are then aggregated into corresponding maintenance costs of the whole superstructure.

To evaluate the LCC, the lifetime costs of each cost category are calculated by using equation 3 and a 3.5\% discount rate. The obtained cost figures are then aggregated in accordance with the cost breakdown structure shown in Figure 2.

\subsection{Superstructure lifecycle analysis}

Four intervention strategies have been investigated:

1. Basic intervention regime: Under this intervention regime all planned inspection and corrective maintenance activities are carried out in accordance to the time schedule. The activities are scheduled for individual assets only in a specific segment of the line.

2. Opportunistic maintenance regime: Under the opportunistic maintenance regime corrective repairs of sleepers can be carried out earlier than planned taking advantage of the arrangements made for the same type of work in an adjacent part of the track. Executing opportunistic maintenance of the assets leads to costs reduction due to reduced planning costs, shorter intervention durations (site preparation is only carried out once) and costs associated with train service interruptions. However, in a long term this strategy may increase the work bank.

3. Concurrent maintenance regime: The first scenario includes the replacement of the whole superstructure when a line possession takes place for the replacement of rails, given other parts of the superstructure are degraded. By carrying out maintenance activities of assets concurrently, cost savings are delivered in the same way as for opportunistic maintenance. 
4. Integrated opportunistic and concurrent maintenance regimes: The last maintenance regime incorporates both opportunistic and concurrent maintenance activities considered in the earlier two maintenance strategies.

The investigation includes analysis of LCC and performance of the railway line over a sixty year period. In order to model infrastructure performance using the superstructure line PN model the model parameter values (i.e. transition parameters, such as delay times or additional marking rules associated with decision making transitions) have been taken from the model presented in [11]. During the simulation of the model, the superstructure performance parameters (model outputs) such as the line downtime due to engineering work, mean time between maintenance activities, duration of speed restrictions and duration of line closures due to poor superstructure condition have been monitored. The performance parameter values obtained and the resulting LCC for each intervention case are presented in Table 2.

Table 2. LCC and Superstructure Performance Results

\begin{tabular}{|l|l|l|l|l|l|}
\hline $\begin{array}{l}\text { Maintenance } \\
\text { Strategy }\end{array}$ & $\begin{array}{l}\text { Downtime, } \\
\text { days }\end{array}$ & $\begin{array}{l}\text { Mean time } \\
\text { between } \\
\text { maintenance, } \\
\text { days }\end{array}$ & $\begin{array}{l}\text { Duration of } \\
\text { speed } \\
\text { restriction, } \\
\text { days }\end{array}$ & $\begin{array}{l}\text { Duration of } \\
\text { line closure, } \\
\text { hours }\end{array}$ & LCC, GBP \\
\hline Basic & 274 & 1.9 & 434 & 0.72 & $7,823,256$ \\
\hline Opportunistic & 230 & 1.7 & 367 & 0.96 & $6,718,355$ \\
\hline Concurrent & 268 & 1.6 & 353 & 0.48 & $7,273,265$ \\
\hline $\begin{array}{l}\text { Opportunistic } \\
\text { and } \\
\text { concurrent }\end{array}$ & 228 & 1.3 & 244 & 0.48 & $6,160,883$ \\
\hline
\end{tabular}

Results demonstrate that for the case study, introducing opportunistic and concurrent maintenance strategies has a positive effect on the predicted LCC, with the most significant reduction (by $21 \%$ ) achieved when combining both the opportunistic and concurrent maintenance strategies. Other performance parameters, except the mean time between maintenance activities, have also been improved as a result of the introduction of the new maintenance strategy. On the other hand, the mean time between activities has decreased slightly. The latter change occurs as a result of an increased number of minor repairs carried out when early signs of degradation are detected. If the train services in the line are not frequent such an intervention strategy would be beneficial in terms of the cost reduction and improvements in infrastructure performance achieved. In the lines where train traffic is intensive and access to the infrastructure for maintenance is limited such an intervention 
strategy may not be appropriate. Even though the total downtime would be shorter, in practice each intervention would last longer as more assets would need to be repaired or replaced simultaneously. In this case the analysis of the impact on train services is required in order to decide whether the proposed maintenance strategies are appropriate. The modelling framework proposed in the paper offers this capability to integrate train operation simulations along the life cycle and LCC analysis of the infrastructure. More details about the enhancement of the modelling framework can be found in [16].

\section{Conclusions}

The paper presents a novel framework for evaluating railway infrastructure LCC, a tool enabling one to make well informed asset management decisions. The framework involves constructing a railway infrastructure performance model for the system in question which is then integrated with a cost model to perform the lifecycle cost analysis. The resulting model provides a monetary criterion in addition to infrastructure performance parameters to enable the evaluation of the range of alternative choices.

The infrastructure performance modelling approach using the PN modelling technique offers a number of advantages:

1. The modular-hierarchical architecture provides a means for configuring the performance model to represent any chosen set of assets in a specified part of the network.

2. The resulting infrastructure model has the capability to predict the performance of individual assets and the infrastructure as a whole system. Furthermore, the performance parameters can be analysed at different network granularity levels, ranging from a single maintainable item to the whole network.

3. By modelling the infrastructure network as a multi-component hierarchical system, interdependencies among different intervention activities (often present in practice), e.g. opportunistic or concurrent maintenance, can be taken into account and their effects on the performance and costs of the whole railway system can be evaluated.

To perform the cost analysis the outputs obtained from the infrastructure performance model are used as input parameters of the cost model. Here a standard LCC model (like that recommended in BS EN 60300) can be used, given it has been adapted to the problem in question. 
An application of the modelling framework has been demonstrated with a case study where superstructure performance over a 60 year period under different intervention strategies was investigated. The infrastructure performance and LCC results for the case study example revealed that combining opportunistic and concurrent maintenance strategies achieves the most significant benefits in terms of cost reductions, shorter overall downtime of the line and a shorter duration of speed restrictions.

Using the information produced by the proposed modelling framework, all potential asset management decisions can be incorporated and policies and regulations formulated delivering the required performance level for the infrastructure subject to the financial constraints. The proposed modelling framework is designed to evaluate the impact of decisions made during the lifetime of the infrastructure. However, asset choices during the design phase and intervention regimes in response to infrastructure degradation during operation phase can also be investigated.

\section{Acknowledgements}

Dr Dovile Rama is the Network Rail Research Fellow in Asset management. John Andrews is the Network Rail Professor of Infrastructure Asset Management. He is also Director of The Lloyd's Register Foundation (LRF) Centre for Risk and Reliability Engineering at the University of Nottingham. They gratefully acknowledge the support of these organizations. (The LRF supports the advancement of engineering-related education, and funds research and development that enhances safety of life at sea, on land and in the air).

\section{References}

1. Duncan, M. Whole Life Costing: completing the analysis: asset investment strategies, business planning and financing for infrastructure companies. Asset Management Conference 2011, IET and IAM, London, UK, 30 November 1 December 2011.

2. Skinner, M., Kirwan, A., Williams, J. Challenges of developing whole life cycle cost models for Network Rail's top 30 assets. Asset Management Conference 2011, IET and IAM, London, UK, 30 November 1 December 2011 
3. Márquez, F. P. G., Lewis, R.W. Tobias, A.M., Roberts, C. Life cycle costs for railway condition monitoring. Transportation Research Part E: Logistics and Transportation Review, Vol. 44, No. 6, 2008; pp. 1175-1187.

4. Andrade, A., Teixeira, P.F. A bi-objective optimization model for maintenance and renewal decisions related to rail track geometry. In Transportation Research Record: Journal of the Transportation Research Board, No. 2261, Transportation Research Board of the National Academies, Washington, D.C., 2011, pp. 163-170.

5. Zhao, J., Chan, A.H.C, Stirling, A.B. and Madelin, K.B. Optimizing Policies of Railway Ballast Tamping and Renewal. In Transportation Research Record: Journal of the Transportation Research Board, No 1943, Transportation Research Board of the National Academies, Washington, D.C., 2006, pp. 50-56.

6. López-Pita, A., Teixeira, P. F., Casas, C., Bachiller, A., Ferreira, P. A. Maintenance Costs of HighSpeed Lines in Europe. In Transportation Research Record: Journal of the Transport Research Board, No 2043, Transportation Research Board of the National Academies, Washington, D.C., 2008, pp. 13-19.

7. Patra, A. P.,Söderholm P., Kumar, U. Uncertainty estimation in railway track life-cycle cost: a case study from Swedish National Rail Administration. Proc. IMechE, Part F: J. Rail and Rapid Transit, Vol. 223, 2009; pp. 285-293.

8. Wenjuan,Z., Wenbin, W., Yi, G. Maintenance strategy optimisation for infrastructure assets through cost modelling. Working Paper. 2013. Coventry, UK: University of Warwick, WBS. http://wrap.warwick.ac.uk/57720/. Accessed May 20, 2014.

9. Murata, T., Petri Nets: Properties, Analysis and Applications, Proceedings of the IEEE, Vol. 77, No. 4, 1989; pp. 541-580.

10. Ajmone Marsan, M., Balbo,G., Conte, G., Donatelli, S., Franceschinis, G. Modelling with Generalized Stochastic Petri Nets. John Wiley \& Sons: 1995.

11. Rama D., Andrews J. A holistic approach to railway infrastructure asset management. International Journal of Performability Engineering, Vol. 11, No. 2, 2015; pp. 107-120.

12. BS EN 60300-3-3. Dependability management. Part 3-3: Application guide - Life cycle costing, 2004. 
13. Andrade, A. R. Renewal decisions from a Life-cycle Cost (LCC) Perspective in Railway Infrastructure: An integrative approach using separate LCC models for rail and ballast components. M. Sc Thesis, Technical University of Lisbon (IST), Lisbon, Portugal, 2008.

14. Network Rail Standard - Track Inspection, NR/L2/TRK/001/mod02, Issue 6, December 2012.

15. Network Rail Standard - Inspection and Maintenance of Permanent Way, NR/L2/TRK/001/mod10, Issue 6, December 2012.

16. Rama D., Andrews J. System-wide assessment of intervention strategies for railway infrastructure. Presented at the 8th International Conference on Modelling in Industrial Maintenance and Reliability (MIMAR). Oxford, UK, 10 - 12 July 2014. 